\title{
Thickness dimensions of the contact layer of soil-rigid body interaction
}

\author{
Bakhtiyar Khusanov ${ }^{*}$ and Barno Rikhsieva \\ IMSS, Academy of Sciences of Rep. of Uzb., 100187 Tashkent, Uzbekistan
}

\begin{abstract}
Solution of the problems of longitudinal interaction of underground pipelines with soil in a one-dimensional statement requires precise measurements of the thickness of the soil layer contacting with the pipeline. In order to determine the dimensions of the contact layer of soil, the behavior of soil medium under shear interaction of a rigid body with soil is studied. Under straining in soil medium, structural changes denoted by the coefficient of structural destruction are taken into account; the condition of complete adhesion is taken in the contact rigid body - soil boundary. The interaction process under consideration is investigated numerically by the finite difference method. The results of the calculations are presented in the form of graphs and are analyzed. From the obtained results, the maximum values of the thickness of soil contact layer are determined as well as the layers with the corresponding degree of structural destruction depending on the geometric dimensions of the rigid body.
\end{abstract}

\section{Introduction}

In theoretical calculations of underground structures on dynamic effects one of the main problems is the correct determination of the reaction force to the structure from soil medium, determined by the conditions of interaction. According to the existing simplified methods of calculation of buildings and structures on dynamic effects, i.e. when considering the wave processes or oscillations of structures only, their interaction with soil are given approximately; this greatly reduces the accuracy of calculations. To improve the accuracy of the problems under consideration, it is necessary to use more adequate equations of state of structure and soil material, and the conditions at the structure - soil contact boundaries. At present, theoretical studies on longitudinal interaction of underground structure with soil using different conditions of interaction are investigated mostly in one-dimensional statement [1-2]. The assumption of one-dimensional theory is confirmed by experimental and theoretical studies [1] for small radii of a steel rod having the Coulomb external dry friction. Note that an account of soil motion in one-dimensional statement leads to the solution of two systems of one-dimensional equations (of soil and structure), the linking aspect is the type of applied boundary conditions (interaction model) at the interaction contact [2-3]. Taking into account the dynamic processes of soil in nonstationary problems [4] has led to a multiple (from two to several dozen times) increase in

\footnotetext{
*Corresponding author: khusanov@mail.ru
} 
stress in the constructions of underground structures compared to the acting loads. One of the main reasons for the multiple increase in stresses is the accepted thickness of the contact layer of soil for one-dimensional calculation. Currently, various models of soil strain have been developed [5-8], which allow the existence of a contact layer of soil [9] and determine their thickness depending on the transverse dimensions of the underground structure. To determine the thickness of the contact layer of soil in the longitudinal interaction there is a need to study the problem of longitudinal interactions of extended rigid bodies with soil in a non-one-dimensional (two-dimensional) statement, which is the purpose of this work.

\section{Statement and method of problem solution}

Consider the process of shear interaction of a rigid (underground) body with soil, taking into account the structural changes in soil, in the case of the un-deformable body motion according to the following law

$$
U_{z 0}=A \sin (2 \pi t / T)
$$

where $A=0,5-1 \mathrm{~m} / \mathrm{s}, T=9 \mathrm{~ms}$. Here soil medium is simulated by the equation

$$
\begin{aligned}
& d P / d t=-K(d V / d t) / V \\
& \frac{d}{d t}\left(\frac{S_{i j}}{G\left(I_{S}\right)}\right)+\lambda \frac{S_{i j}}{G_{*}}=2 \frac{d e_{i j}}{d t},
\end{aligned}
$$

in the case of unloading

$$
d S_{i j} / d t=2 G_{*} \exp \left(\alpha_{1}\right) d e_{i j} / d t
$$

where $\alpha_{1}$ is the coefficient characterizing the degree of changes in shear modulus under unloading and subsequent loading, and depending on the loading history and on $I_{S}^{\prime} ; I_{S}^{\prime}$ is the value of the degree of soil destruction at the beginning of unloading; the function of soil plasticity is:

$$
Y(P)=Y_{0}+\frac{\mu P}{1+\mu P /\left(Y_{p l}-Y_{0}\right)} .
$$

The parameters and their values are given in [5-8].

So, the problem is reduced to the solution of the basic equations:

$$
\rho \frac{d U_{r}}{d t}=\frac{\partial \sigma_{r r}}{\partial r}+\frac{\partial \tau_{r z}}{\partial z}+\frac{\sigma_{r r}-\sigma_{\varphi \varphi}}{r}, \quad \rho \frac{d U_{z}}{d t}=\frac{\partial \sigma_{z z}}{\partial z}+\frac{\partial \tau_{r z}}{\partial r}+\frac{\tau_{r z}}{r},
$$

where $z$ is the axis of a cylindrical body; $r$ is the radial coordinate. Here the components of the stress tensor are related to the corresponding components of stress deviators by the relationships (2)-(3), which have the form:

$$
\sigma_{r}=S_{r}-P, \quad \sigma_{z z}=S_{z z}-P, \quad \sigma_{\varphi \varphi}=S_{\varphi \varphi}-P, \quad \tau_{r z}=S_{r z},
$$

Continuity equation is 


$$
\partial U_{r} / \partial r+\partial U_{z} / \partial z+U_{r} / r=\dot{V} / V
$$

where $V=\rho_{0} / \rho$ is the relative volume; $\dot{V}=d V / d t ; \rho_{0}$ is the initial density of the medium;

and relationships

$$
\begin{gathered}
\dot{\varepsilon}_{r r}=\partial U_{r} / \partial r, \quad \dot{\varepsilon}_{z z}=\partial U_{z} / \partial z, \quad \dot{\varepsilon}_{\varphi \varphi}=U_{r} / r \\
\dot{\varepsilon}_{r z}=\left(\partial U_{r} / \partial r+\partial U_{z} / \partial z\right) / 2 .
\end{gathered}
$$

together with (2)-(4) with zero initial and boundary (1) conditions on the contact surface.

The problem is solved by the finite difference method using the Wilkins scheme for two-dimensional axisymmetric problems. Note that this scheme has a second order of accuracy. The finite-difference relations of the considered equations are available in [10].

\section{Results and their analysis}

Determine the effect of geometrical dimensions of a rigid body, the parameters of its motion, mechanical and strength characteristics of soil on the thickness of soil contact layer. To do this, the dependence obtained by the solution of the problem [9] is used. The options for different values of the rigid body radius are shown in Table 1.

Table 1. Options of calculation

\begin{tabular}{|c|c|c|c|c|c|}
\hline Optionts No & $\boldsymbol{A}, \mathbf{m} / \mathbf{s e c}$ & $\boldsymbol{T}, \mathbf{s e c}$ & $\boldsymbol{E} *, \mathbf{G P a}$ & $\boldsymbol{Y}_{\mathbf{0}}$ & $\mathbf{\alpha}=\mathbf{a}_{\mathbf{0}}+\mathbf{\alpha}^{*}$ \\
\hline 1 & 0,5 & 0,009 & 1,6 & $G^{*} / 500$ & $1+1$ \\
\hline 2 & 0,5 & 0,009 & 1,6 & $G^{*} / 500$ & $1+2$ \\
\hline 3 & 0,5 & 0,009 & 1,6 & $G^{*} / 1000$ & $1+1$ \\
\hline 4 & 1,5 & 0,009 & 1,6 & $G^{*} / 500$ & $1+1$ \\
\hline 5 & 0,5 & 0,009 & 1,2 & $G^{*} / 500$ & $1+1$ \\
\hline 6 & 0,5 & 0,012 & 1,6 & $G^{*} / 500$ & $1+1$ \\
\hline 7 & 0,5 & 0,004 & 1,6 & $G^{*} / 500$ & $1+1$ \\
\hline
\end{tabular}

Figures 1-5 show the changes in maximum value of the degree of structural destruction of soil depending on the distance from a contact surface. Curves 1-8 in these figures correspond to the radii of a cylindrical underground body and equal to $R_{0}=0.01 \mathrm{~m} ; 0.1 \mathrm{~m}$; $0.2 \mathrm{~m} ; 0.5 \mathrm{~m} ; 1 \mathrm{~m} ; 2 \mathrm{~m} ; 5 \mathrm{~m}$ and $10 \mathrm{~m}$, respectively. Fig. 1 corresponds to the calculation of option No. 1. This figure shows that the complete structural destruction of soil occurs directly near the underground body; with the distance from the contact surface the values of $I_{S \max }$ from the unit are reduced. When $I_{S \max }<1, I_{S \max }$ is the corresponding degree of soil destruction (incomplete structural destruction). At $R_{0}=0,01 \mathrm{~m}$ (curve 1) the degree of structural disturbance of soil decreases intensively with the distance from the boundary of complete structural destruction. With increasing radius of the underground body, as seen in Fig.1, the value of the thickness of soil layer under complete structural destruction increases.

The layer of soil corresponding to the complete structural destruction (disturbance) can be considered as a contact layer of soil in underground rigid body - soil interaction. The thickness of the contact layer of soil, depending on the radius of the underground body, in 
the problem under consideration, ranges from $6 \mathrm{~cm}$ (for $R_{0}=0.01 \mathrm{~m}$, corresponding to underground pipelines) to $50 \mathrm{~cm}$ (for $R_{0}=10 \mathrm{~m}$, corresponding to metro tunnels)

a)

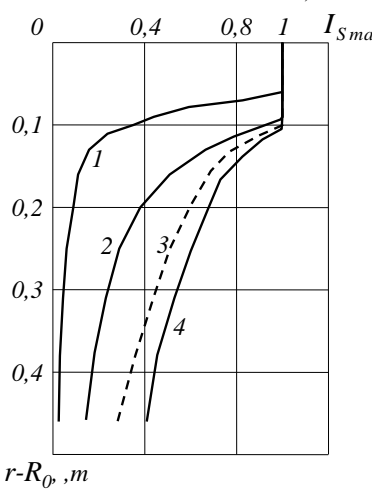

b)

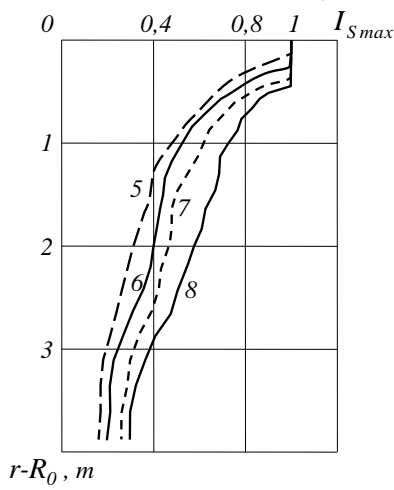

Fig. 1. Degree of structural destruction of soil with the distance from the contact surface at different diameters of underground body

From the results of the previous problem, it can be assumed that the values of the thickness of the soil contact layer can be significantly influenced by the coefficient $\alpha$ characterizing the degree of structural destruction of soil, by the limit value of the structural strength of soil to shear (yield strength), by the values of mechanical characteristics of the medium and the parameters of motion of the underground body interacting with soil. Changes in the degree of structural destruction of soil with a distance in the case of changes in these parameters are shown in Figs. 2-5.

a)

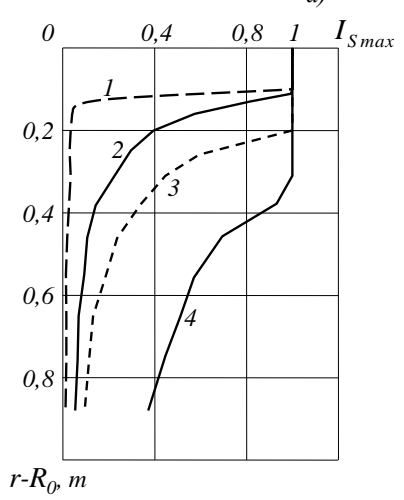

b)

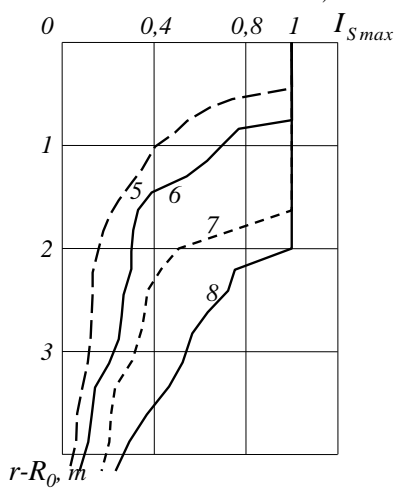

Fig. 2. Degree of structural destruction of soil with the distance from the contact surface at $\alpha=3$

As seen from Fig. 2, an increase in initial value of $\alpha=\alpha_{0}+\alpha_{*}$ leads to a significant increase in the thickness of the contact layer of soil (option 2). In this case, an increase in the thickness of the contact layer of soil increases, mainly with increasing radius of the underground body. In particular, at $R_{0}=10 \mathrm{~m}$ the thickness reaches two meters, i.e. increases by about 4 times compared to the result of the first option (Fig.1). The thickness of the contact layer of soil also increases at decreasing values of $Y_{0}$ (option 3, Fig.3) or at 
increasing initial value of $E_{*}$. Reducing initial value of $E_{*}$ significantly reduces the thickness of the contact layer of soil only at large radii of the underground body (option 5 , Fig.4).
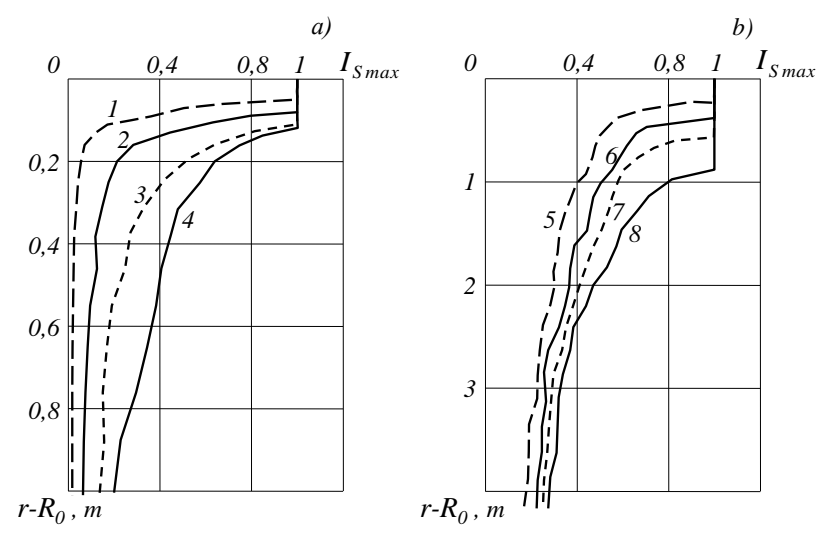

Fig. 3. Degree of structural destruction of soil with the distance from the contact surface at $\mathrm{Y}_{0}=\mathrm{G}_{*} / 1000$
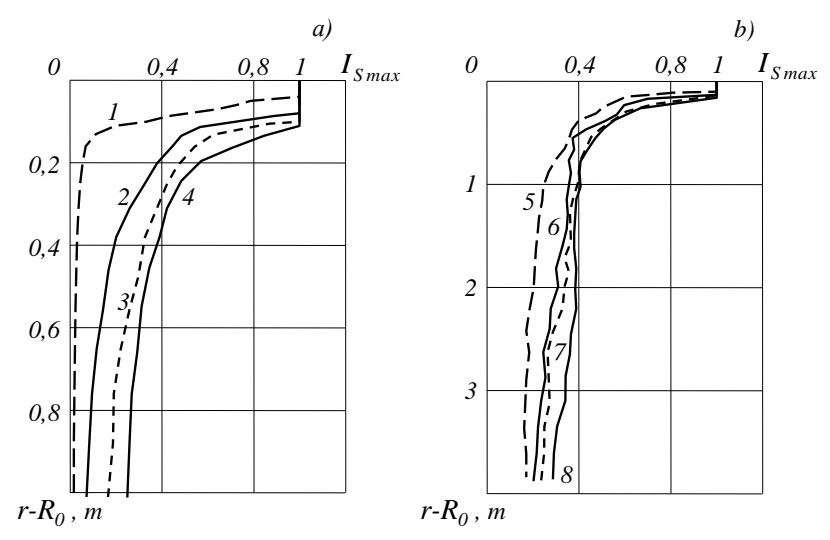

Fig. 4. Degree of structural destruction of soil with the distance from the contact surface at $\mathrm{E}_{*}=1,2 \mathrm{GPa}$

The calculations show that within the considered options (options 4,6 and 7), the change in the parameters of the underground body motion does not significantly affect the values of the thickness of the contact layer of soil (Fig.5 corresponds to option 4). Changes in the values of the thickness of the contact layer of soil depending on the radius of the underground body are shown in Fig. 6.

From Fig. 6 it is seen that the thickness function of the contact layer depending on the dimensions of the underground body is nonlinear. At small values of the radius of the underground body, it is approximately logarithmic. In Fig.6, such a change in the thickness of the contact layer of soil from the radius of a rigid body is given on the logarithmic grid. Here we can see the fact that the thickness of the layer is proportional to the logarithmic function of the radius of the body at small values of the latter (up to 1 meter for the radius of the underground structure). Nonlinearity is clearly expressed at large radii of the underground body. 


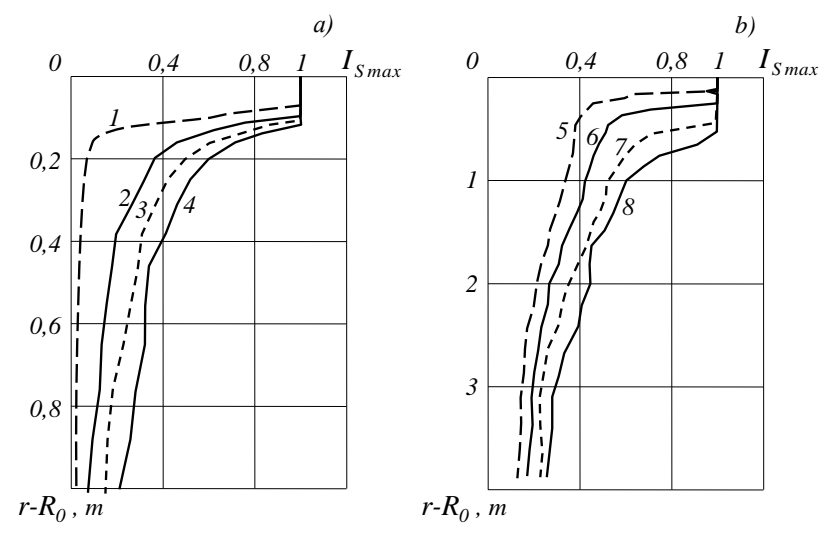

Fig. 5. Degree of structural destruction of soil with the distance from the contact surface at $A=1,5 \mathrm{~m} / \mathrm{s}$
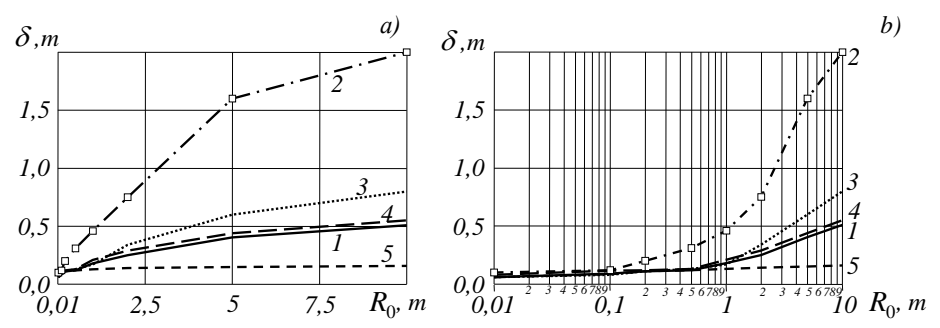

Fig. 6. Dimensions of the thickness of the contact layer of soil depending on the radius of underground structure

From Fig.6 it can be seen that the function of the thickness of the contact layer depending on the dimensions of the underground body is nonlinear. For small values of the radius of the underground body, it is approximately logarithmic. In Fig.6, such a change in the thickness of the contact layer of the soil from the radius of the rigid body is given on the logarithmic grid. Here we can see that the thickness of the layer is proportional to the logarithmic function of the radius of the body at small values of the latter (up to 1 meter for the radius of the underground structure). Nonlinearity is clearly expressed at large radii of the underground body.

As seen from the results of studies to determine the thickness of the contact layer of soil for a rigid body having small cross-sections (under $50 \mathrm{~cm}$ in radius) at different values of effective load, mechanical and strength characteristics of soil, the thickness of the layer subjected to complete structural destruction does not exceed the transverse dimension.

\section{Conclusion}

According to the results of problem solution, the existence of the contact layer of soil is revealed and the values of the thickness of the contact layer of soil are determined depending on the dimensions of the interacting rigid body. The results of one-dimensional problems [1-3] with sufficient accuracy coincide with experimental observations. In [4] for an underground pipeline with an external diameter $0.2 \mathrm{~m}$, a one-dimensional motion of the soil layer with a diameter of one meter has been adopted, so in these calculations a multiple increase in stresses in the underground structure is observed. 
The research is conducted under financial support of the Ministry of Innovation Development of the Republic of Uzbekistan on the project FA-F4-F003.

\section{References}

1. K.S. Sultanov, Wave theory of seismic stability of underground structures (Tashkent, Fan, 2016)

2. K.S. Sultanov, Int. J. Appl. Mech. 3, 60 (1993)

3. K.S. Sultanov, J. Appl. Mech. \& Tech. Phys. 1, 40 (1993)

4. K.S. Sultanov, Z.S. Makhmudov, Uzb. J. Prob. Mech. 6, 16 (1994)

5. B.E. Khusanov, Dokl. Acad. Sci. 2, 35 (2004)

6. K.S. Sultanov, B.E. Khusanov, Uzb. J. Prob. Mech. 4, 16 (2000)

7. K.S. Sultanov, B.E. Khusanov, Soil Mech. \& Found. Eng. 3, 80 (2001)

8. B.E.Khusanov, XI All-Russian Congress on Fundamental Problems of Theoretical and Applied Mechanics, Collection of reports (Kazan, University Press, 2015)

9. B.E.Khusanov, Proceedings Conf. of Mechanics of Deformable Rigid Body (Tashkent, TIIAME, 2018)

10. M.L. Wilkins, Computer Simulation of Dynamic Phenomena (Berlin, Springer-Verlag, 2010) 